\title{
SELEÇÃO DE PARÂMETROS ATRAVÉS DO MÉTODO DE TAGUCHI PARA SOLDAGEM DA LIGA DE ALUMÍNIO 5052H32 PELO PROCESSO MIG
}

\author{
D.T. ALMEIDA ${ }^{1}$, J.H.C. SOUZA, M.R.K. KUNTZ, S.R. KNEBELKAMP \\ Universidade Federal do Rio Grande do Sul \\ diegot@bruning.com.br ${ }^{1}$
}

Artigo submetido em 22/03/2018 e aceito em 09/08/2019

DOI: $10.15628 /$ holos.2019.7121

\begin{abstract}
RESUMO
É crescente a utilização do alumínio e suas ligas nos mais diversos setores da indústria automotiva. Sendo, o processo de soldagem MIG é amplamente utilizado devido às diversas vantagens, como grande versatilidade, diversidade de posições de soldagem e elevada velocidade de soldagem. Neste trabalho, utilizou-se o método de Taguchi para otimizar o número de experimentos, a fim de obter a influência dos fatores de controle sobre as variáveis respostas e determinar as condições ideais para aplicação da liga $5052 \mathrm{H} 32$ na soldagem através do processo MIG com transferência metálica por curto-circuito. Para realização dos ensaios, realizou-se a soldagem dos corpos de prova na posição
\end{abstract}

plana, variando os seguintes parâmetros: o tipo de metal de adição, o tipo de gás de proteção, a velocidade de soldagem, a vazão do gás de proteção e o stick-out. Foi utilizado como matriz ortogonal o design L36 (matriz ortogonal de design misto). Por sua vez, as variáveis respostas escolhidas foram: taxa de deposição, aporte térmico e inspeção visual. A combinação dos níveis de controle apontados como ótimos pelo método de Taguchi resultaram em valores para as variáveis respostas consideradas adequadas para a soldagem MIG, sendo que o parâmetro de maior influência para otimização do processo e qualidade de soldagem é a velocidade de soldagem.

PALAVRAS-CHAVE: Processo de soldagem MIG, Método de Taguchi, Liga de alumínio, Taxa de deposição, Aporte térmico.

\section{USING THE TAGUCHI METHOD TO SELECT WELDING PARAMETERS FOR WELDING OF ALUMINUM ALLOY 5052H32 THROUGH THE GMAW PROCESS}

\begin{abstract}
The use of aluminum and its alloys in the most diverse sectors of the automotive industry. Given these needs, the MIG welding process is widely used because of the many advantages such as high versatility, welding position diversity and high welding speed. In this work, the Taguchi method was used to optimize the number of experiments in order to obtain the influence of the control factors on the response variables and to determine the ideal conditions for $5052 \mathrm{H} 32$ alloy application in the MIG process with metallic transfer By short circuit. In order to carry out the tests, the welding of the test pieces was carried out in the flat position,
\end{abstract}

varying the following parameters: the type of addition metal, the type of protection gas, the welding speed, Protection gas and the stick-out. The orthogonal matrix L36 (orthogonal matrix of mixed design). In turn, the variables chosen were: deposition rate, thermal input and visual inspection. The combination of the control levels indicated as optimal by the Taguchi method resulted in values for the responses considered adequate for MIG welding, and the parameter of greatest influence for process optimization and welding quality is the welding speed.

KEYWORDS: Welding process GMAW, Taguchi method, Aluminum alloy, Deposition rate, Heat input 


\section{INTRODUÇÃO}

A soldagem é um dos mais importantes processos industriais de fabricação de peças metálicas. O sucesso da soldagem está associado a diversos fatores e, em particular, com a sua relativa simplicidade operacional (LIAO e CHEN, 1998).

O processo de soldagem MIG (Metal Inert Gas), consiste em um aquecimento localizado da região a se unir, até que atinja o ponto de fusão, formando-se então a poça de metal líquido, que receberá o metal de adição também na forma fundida. A energia necessária para fundir tanto o metal de base quanto o metal de adição, é fornecida pelo arco elétrico (MACHADO, 1996). No processo MIG o arame/eletrodo é sempre o polo positivo do arco elétrico (ALCAN, 2017).

Vários são os parâmetros que possuem influência na qualidade da junta soldada, sendo que para produzir soldas com um bom desempenho, é preciso conhecer a combinação de parâmetros adequados para obtenção de juntas soldadas de boa qualidade com o mínimo de defeitos (BOHRER, 2013).

A velocidade de soldagem tem influência direta na quantidade de calor que é cedido a peça. Aumentando a velocidade, altera-se a geometria do cordão de solda, diminuindo a penetração, a largura e o reforço do cordão. A diminuição excessiva da velocidade de soldagem por sua vez, pode ocasionar problemas metalúrgicos, devido à elevada energia de soldagem, além de impactar negativamente na produtividade (MARQUES et al., 2009).

Para Wainer et al. (1992) a tensão de soldagem está diretamente associada ao comprimento do arco, ou seja, uma tensão baixa exige um pequeno comprimento do arco, consequentemente, uma pequena distância da ponta do arame até a poça de fusão. Sendo assim, a seleção da tensão deve ocorrer de acordo com a intensidade da corrente, gás de proteção, tipo de arame, além da geometria do cordão que se deseja obter. Sendo que, a distância do eletrodo (stick-out) possui influência direta no aumento ou queda de tensão.

Marques et al. (2009), destacam que o tipo de gás de proteção influencia também na penetração, largura do cordão, velocidade máxima de soldagem, tendência de mordeduras e o custo do processo.

A correta vazão do gás de proteção, estabiliza o arco, controlam a operacionalidade e a transferência do metal, possuem influência sobre a geometria do cordão de solda, propriedades mecânicas e características metalúrgicas da solda. Assim, se torna importante a escolha de uma correta vazão do gás de proteção para prevenir eventuais defeitos na soldagem (MARQUES, et al., 2009).

Para se obter um aumento da qualidade de soldagem é necessário um conhecimento a respeito dos parâmetros e variáveis que afetam o processo. Assim, o objetivo desta pesquisa é o de melhorar as características do processo de soldagem MIG, através da identificação e ajuste dos seus fatores controláveis, que irão minimizar a variação do produto final, ajustando os fatores no seu nível ótimo. Assim, garantimos que os produtos possam ser fabricados de maneira que se tornem mais robustos a toda e qualquer mudança que possa ocorrer e que seja incontrolável (condições ambientais, variação de temperatura, tempo de acondicionamento, etc.). 
Para isto, utilizamos o método de Taguchi para avaliar a influência de algumas das variáveis de soldagem, denominadas neste trabalho como fatores controláveis, sobre as características dos cordões de solda (variáveis respostas) e determinar as condições ideais para aplicação da soldagem MIG. Cinco parâmetros foram utilizados neste trabalho: o tipo de metal de adição (arame de solda), o tipo de gás de proteção, a velocidade de soldagem, a vazão do gás de proteção e o stick-out (distância do bico de contato da peça - DBCP). O trabalho foi desenvolvido no departamento de P\&D da empresa Bruning Tecnometal, a fim de encontrar o parâmetro ideal para garantir soldas de boa qualidade com o mínimo de defeitos.

\section{MATERIAIS E MÉTODOS}

Neste estudo, foi realizada a solda de chapas com 2,50mm de espessura da liga 5052H32, com dimensão de $150 \mathrm{~mm} \times 300 \mathrm{~mm}$. A Tabela 1 apresenta a composição química e propriedades mecânicas do material utilizado no experimento.

Tabela 1 - Composição química e propriedades mecânicas da liga Al5052H32.

\begin{tabular}{|c|c|}
\hline \multicolumn{2}{|c|}{ Composição Química } \\
\hline Manganês- Mn(\%) & $0.20-0.50$ \\
\hline Silício - Si (\%) & 0.20 MÁX. \\
\hline Cobre - Cu (\%) & 0.15 MÁX. \\
\hline Titânio - Ti (\%) & 0.10 MÁX. \\
\hline Cromo - Cr (\%) & 0.10 MÁX. \\
\hline Magnésio-Mg(\%) & $4.0-5.0$ \\
\hline Zinco-Zn(\%) & 0.25 MÁX. \\
\hline Ferro-Fe(\%) & 0.35 MÁX. \\
\hline \multicolumn{2}{|c|}{ Propriedades Mecânicas } \\
\hline Limite Escoamento $\left(\mathrm{N} / \mathrm{mm}^{2}\right)$ & 160 MíN. \\
\hline Limite Resist. Tração (N/mm²) & $215-265$ \\
\hline Along. (\%) & 7 MíN. \\
\hline
\end{tabular}

Fonte: Adaptada da Norma ABNT NBR 6834 e NBR 7823

A soldagem dos corpos de prova foi através do processo MIG, realizadas de forma mecanizada na posição plana. Foi utilizado um dispositivo mecanizado de movimentação linear com velocidade constante como meio de obter juntas soldadas com propriedades uniformes e com boa repetitividade. Juntamente com o dispositivo foi utilizada uma fonte de soldagem modelo Trans Puls Synergic 5000MV da marca Fronius. Os resultados de corrente, tensão e tempo de arco aberto, foram obtidos através do sistema de aquisição de dados Fronius Xplorer RCU5000. Na Figura 1 é possível observar o dispositivo mecanizado empregado na soldagem MIG. 


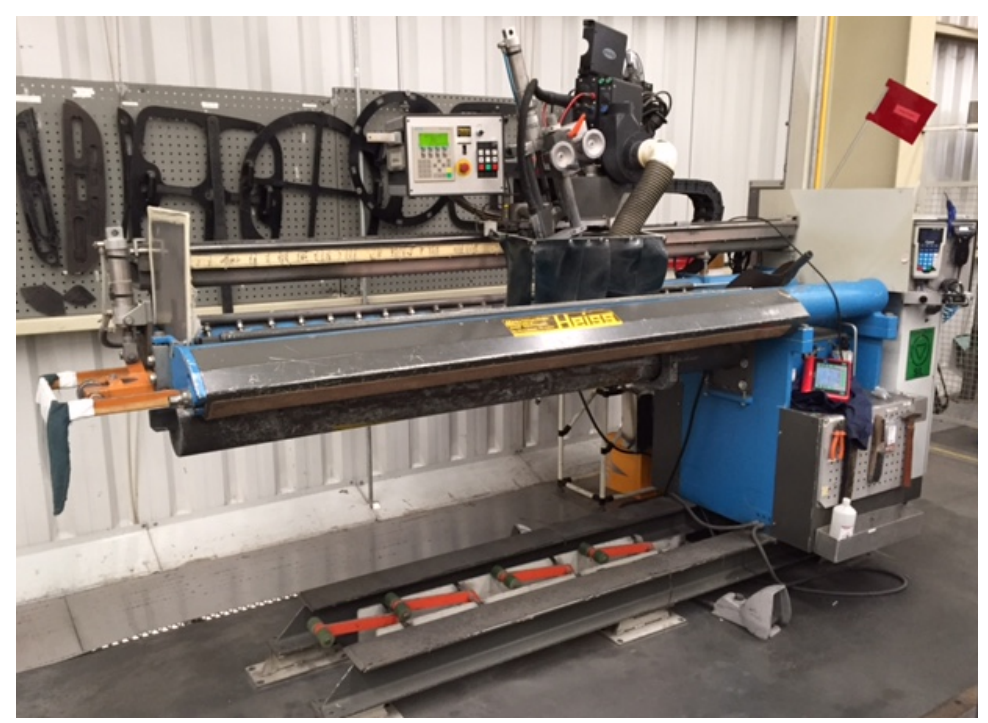

Figura 1 - Aparato utilizado no processo de soldagem MIG

Para a realização do experimento, primeiramente foram identificados os parâmetros de maior influência no processo de soldagem MIG. Cinco parâmetros foram escolhidos: o tipo de metal de adição (arame de solda), o tipo de gás de proteção, a velocidade de soldagem, a vazão do gás de proteção e o stick-out.

A fim de encontrar a combinação ideal dos parâmetros de soldagem e compreender a importância de cada parâmetro na otimização da soldagem MIG foi implementado o Método de Taguchi usando o software Minitab, versão Free Trial. Foi utilizada como matriz ortogonal o design L36 (matriz ortogonal de design misto) que permite estudar todo o intervalo dos parâmetros do processo com o menor número de experimentos possíveis, sendo três fatores de 3-níveis e dois fatores de 2-níveis. Todos os cincos parâmetros do processo já especificados, foram selecionados como variáveis do D.O.E (Design of Experiment). A Tabela 2 resume os fatores utilizados neste estudo.

Tabela 2 - Parâmetros do processo de soldagem.

\begin{tabular}{llll}
\hline Fator & \multicolumn{3}{c}{ Níveis de Valores } \\
& Nível 1 & Nível 2 & Nível 3 \\
\hline Arame de Solda & ER5183 & ER5356 & - \\
Gás de Proteção & $100 \% \mathrm{Ar}$ & $75 \% \mathrm{Ar+25 \% He}$ & - \\
Vazão do Gás [L/min.] & 18 & 22 & 25 \\
Velocidade de Soldagem [mm/min.] & 1000 & 1300 & 1600 \\
Stick-Out [mm] & 10 & 15 & 20 \\
\hline
\end{tabular}

A composição química dos dois tipos de arame de solda, o ER5183 e o ER5356, podem ser visualizadas na Tabela 3, a mesma segue as características informadas pela norma AWS 5.10.

Tabela 3 - Composição química dos arames de solda.

\begin{tabular}{ccccccc}
\hline AWS & Si (\%) & Fe (\%) & $\mathrm{Cu}(\%)$ & $\mathrm{Mn}(\%)$ & $\mathrm{Mg}(\%)$ & $\mathrm{Cr}(\%)$ \\
ER-5183 & 0,4 & 0,4 & 0,1 & $0,5-1,0$ & $4,3-5,2$ & $0,05-0,25$ \\
ER-5356 & 0,25 & 0,4 & 0,1 & $0,05-0,2$ & $4,5-5,5$ & $0,05-0,2$ \\
\hline
\end{tabular}

Obs.: quando não se menciona uma faixa, a porcentagem é máxima.

Fonte: Adaptada da norma AWS 5.10. 
As chapas foram soldadas em temperatura ambiente, sem pré ou pós-aquecimento do material, depois de soldadas foram visualmente inspecionadas. A Tabela 4 apresenta todas as condições experimentais definidas pelo Método de Taguchi. Sendo que cada uma das 36 combinações produziu 3 repetições dos corpos de prova, totalizando 108 chapas soldadas.

Tabela 4 - Condições experimentais através do Método de Taguchi

\begin{tabular}{|c|c|c|c|c|c|}
\hline $\begin{array}{c}\text { Amostra } \\
\#\end{array}$ & $\begin{array}{c}\text { Arame de } \\
-\end{array}$ & $\begin{array}{c}\text { Gás de Proteção } \\
-\end{array}$ & $\begin{array}{l}\text { Vazão do } \\
\text { [L/min.] }\end{array}$ & $\begin{array}{l}\text { Velocidade de } \\
\text { [mm/min.] }\end{array}$ & $\begin{array}{l}\text { Stick-out } \\
\text { [mm] }\end{array}$ \\
\hline 1 & ER5183 & $100 \% \mathrm{Ar}$ & 18 & 1000 & 10 \\
\hline 2 & ER5183 & $100 \% \mathrm{Ar}$ & 22 & 1300 & 15 \\
\hline 3 & ER5183 & $100 \% \mathrm{Ar}$ & 25 & 1600 & 20 \\
\hline 4 & ER5183 & $100 \% \mathrm{Ar}$ & 18 & 1000 & 10 \\
\hline 5 & ER5183 & $100 \% \mathrm{Ar}$ & 22 & 1300 & 15 \\
\hline 6 & ER5183 & $100 \% \mathrm{Ar}$ & 25 & 1600 & 20 \\
\hline 7 & ER5183 & $100 \% \mathrm{Ar}$ & 18 & 1000 & 15 \\
\hline 8 & ER5183 & $100 \% \mathrm{Ar}$ & 22 & 1300 & 20 \\
\hline 9 & ER5183 & $100 \% \mathrm{Ar}$ & 25 & 1600 & 10 \\
\hline 10 & ER5183 & $75 \% \mathrm{Ar}+25 \% \mathrm{He}$ & 18 & 1000 & 20 \\
\hline 11 & ER5183 & $75 \% \mathrm{Ar}+25 \% \mathrm{He}$ & 22 & 1300 & 10 \\
\hline 12 & ER5183 & $75 \% \mathrm{Ar}+25 \% \mathrm{He}$ & 25 & 1600 & 15 \\
\hline 13 & ER5183 & $75 \% \mathrm{Ar}+25 \% \mathrm{He}$ & 18 & 1300 & 20 \\
\hline 14 & ER5183 & $75 \% \mathrm{Ar}+25 \% \mathrm{He}$ & 22 & 1600 & 10 \\
\hline 15 & ER5183 & $75 \% \mathrm{Ar}+25 \% \mathrm{He}$ & 25 & 1000 & 15 \\
\hline 16 & ER5183 & $75 \% \mathrm{Ar}+25 \% \mathrm{He}$ & 18 & 1300 & 20 \\
\hline 17 & ER5183 & $75 \% \mathrm{Ar}+25 \% \mathrm{He}$ & 22 & 1600 & 10 \\
\hline 18 & ER5183 & $75 \% \mathrm{Ar}+25 \% \mathrm{He}$ & 25 & 1000 & 15 \\
\hline 19 & ER5356 & $100 \% \mathrm{Ar}$ & 18 & 1300 & 10 \\
\hline 20 & ER5356 & $100 \% \mathrm{Ar}$ & 22 & 1600 & 15 \\
\hline 21 & ER5356 & $100 \% \mathrm{Ar}$ & 25 & 1000 & 20 \\
\hline 22 & ER5356 & $100 \% \mathrm{Ar}$ & 18 & 1300 & 15 \\
\hline 23 & ER5356 & $100 \% \mathrm{Ar}$ & 22 & 1600 & 20 \\
\hline 24 & ER5356 & $100 \% \mathrm{Ar}$ & 25 & 1000 & 10 \\
\hline 25 & ER5356 & $100 \% \mathrm{Ar}$ & 18 & 1600 & 15 \\
\hline 26 & ER5356 & $100 \% \mathrm{Ar}$ & 22 & 1000 & 20 \\
\hline 27 & ER5356 & $100 \% \mathrm{Ar}$ & 25 & 1300 & 10 \\
\hline 28 & ER5356 & $75 \% \mathrm{Ar}+25 \% \mathrm{He}$ & 18 & 1600 & 15 \\
\hline 29 & ER5356 & $75 \% \mathrm{Ar}+25 \% \mathrm{He}$ & 22 & 1000 & 20 \\
\hline 30 & ER5356 & $75 \% \mathrm{Ar}+25 \% \mathrm{He}$ & 25 & 1300 & 10 \\
\hline 31 & ER5356 & $75 \% \mathrm{Ar}+25 \% \mathrm{He}$ & 18 & 1600 & 20 \\
\hline 32 & ER5356 & $75 \% \mathrm{Ar}+25 \% \mathrm{He}$ & 22 & 1000 & 10 \\
\hline 33 & ER5356 & $75 \% \mathrm{Ar}+25 \% \mathrm{He}$ & 25 & 1300 & 15 \\
\hline 34 & ER5356 & $75 \% \mathrm{Ar}+25 \% \mathrm{He}$ & 18 & 1600 & 10 \\
\hline 35 & ER5356 & $75 \% \mathrm{Ar}+25 \% \mathrm{He}$ & 22 & 1000 & 15 \\
\hline 36 & ER5356 & $75 \% \mathrm{Ar}+25 \% \mathrm{He}$ & 25 & 1300 & 20 \\
\hline
\end{tabular}

As juntas soldadas não receberam nenhum tratamento em especial, somente a limpeza com álcool isopropílico, essa limpeza teve como objetivo a retirada de óleo e sujidades inerentes do processo de corte dos corpos de prova que poderiam vir a interferir nos resultados. Inicialmente, as amostras foram pesadas individualmente em uma balança de precisão de 1 grama, os dados foram arquivados para posterior análise da taxa de deposição do arame. 
A fim de avaliar a aparência do cordão de solda, todas as amostras foram submetidas a uma análise de inspeção, onde foram caracterizadas através de um padrão de cores conforme Figura 2.

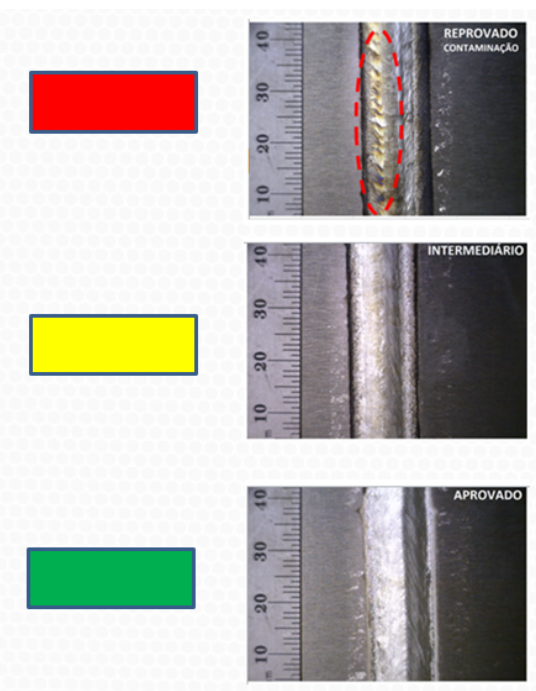

Figura 2 - Caracterização dos defeitos através de inspeção visual.

Está análise traz referência a um código de cores, da seguinte maneira: a cor vermelha indica falhas ocorridas com intensidade elevada, podendo ser pela contaminação do cordão ou pela existência de porosidade superficial, a cor amarela com intensidade moderada e a cor verde com intensidade baixa ou ausência de problemas.

A taxa de deposição de arame é a relação entre a massa final e a massa inicial de uma peça soldada com o tempo de arco aberto necessário para soldar a peça (GARCIA, 2011). É calculado através da seguinte equação:

$$
T D=\left(\frac{M f-M i}{t}\right)
$$

Onde:

$$
\begin{aligned}
& \text { TD }=\text { taxa de deposição do arame }[\mathrm{kg} / \mathrm{h}] \\
& \mathrm{M} f=\text { massa final do corpo de prova }[\mathrm{g}] \\
& \mathrm{M} i=\text { massa inicial do corpo de prova }[\mathrm{g}] \\
& t=\text { tempo de arco aberto }[\mathrm{h}]
\end{aligned}
$$

Com os valores de massa inicial e final já coletados, os resultados de tempo de arco aberto foram obtidos através do sistema de aquisição de dados Fronius Xplorer RCU5000, obtendo o tempo exato de soldagem de cada corpo de prova.

O método de cálculo para o aporte térmico é descrito pela norma DIN EN 1011-1 (2013) e apresentada pela Equação 2.

$$
\mathrm{AT}=\frac{U \cdot I \cdot 60}{v s \cdot 1000} \cdot k=\left[\frac{k J}{\mathrm{~mm}}\right]
$$


Onde:

$\mathrm{U}=$ tensão de soldagem $[\mathrm{V}]$

$\mathrm{I}=$ corrente de soldagem $[\mathrm{A}]$

vs $=$ velocidade de soldagem $[\mathrm{mm} / \mathrm{min}]$

$\mathrm{k}$ = eficiência térmica (conforme DIN EN 1011-1:2013)

De acordo com Búrigo et al. (2016) o calor é a principal fonte de energia dos processos de soldagem, sendo necessária a quantidade e intensidade corretas para se garantir a boa qualidade da junta soldada. Este calor fornecido influencia diretamente as transformações metalúrgicas e fenômenos mecânicos que ocorrem no material, em consequência dos ciclos térmicos e das temperaturas submetidas.

\section{RESULTADOS}

Neste item é descrito diversos aspectos da influência dos parâmetros de soldagem sobre as características das soldas produzidas pelo processo MIG, principalmente por meios da confrontação dos resultados obtidos pelos diversos testes realizados. Uma das grandes vantagens da condução de experimentos usando o método de Taguchi é a previsão da combinação que fornece uma resposta ótima de cada fator de controle, desde que não haja forte interação entre os efeitos dos fatores de controle sobre as respostas. A Figura 3 apresenta os resultados referentes a inspeção visual, considerando a razão sinal-ruído como maior é melhor.

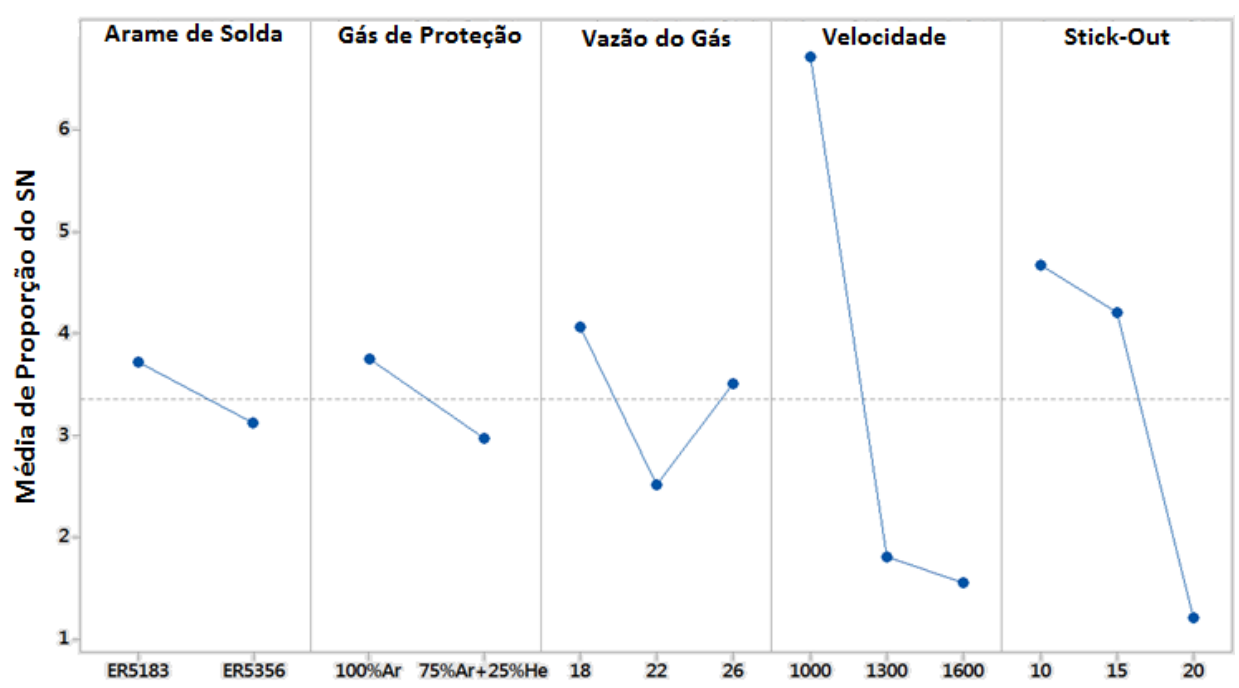

Figura 3 - Razão S/N da qualidade superficial do cordão de solda

A Figura 3 mostra a influência dos fatores de controle sobre a qualidade superficial do cordão de solda. A combinação dos cinco melhores níveis de cada parâmetro que otimizam o processo de soldagem MIG com base nos resultados experimentais para maximizar a variável resposta é apresentada na Tabela 5.

Tabela 5 - Melhor configuração de parâmetro de soldagem para a qualidade superficial.

\begin{tabular}{c|c|c|c|c}
\hline $\begin{array}{c}\text { Arame de Solda } \\
-\end{array}$ & $\begin{array}{c}\text { Gás de Proteção } \\
-\end{array}$ & $\begin{array}{c}\text { Vazão do Gás } \\
{[\mathrm{L} / \mathrm{min} .]}\end{array}$ & $\begin{array}{c}\text { Velocidade de Soldagem } \\
{[\mathrm{mm} / \mathrm{min} .]}\end{array}$ & $\begin{array}{c}\text { Stick-out } \\
{[\mathrm{mm}]}\end{array}$ \\
\hline ER5183 & 100\%Ar & 18 & 1000 & 10 \\
\hline
\end{tabular}


Através de uma análise conjunta da Figura 3 e da Tabela 5 obtêm-se as seguintes informações da aplicação do método de Taguchi com relação à influência dos fatores de controle sobre a qualidade superficial. A maior variação do sinal-ruído para a qualidade superficial do cordão pode ser verificada pela velocidade de soldagem, mostrando que este é o parâmetro com maior influência sobre esta característica. A ordem em que cada parâmetro selecionado neste estudo afeta a qualidade superficial pode ser classificada como: velocidade de soldagem > stick-out > vazão do gás > gás de proteção > arame de solda. A velocidade de soldagem em $1000 \mathrm{~mm} / \mathrm{min}$ possui a melhor resposta para razão sinal-ruído. Também é valido citar o parâmetro de stick-out com $10 \mathrm{~mm}$ mostrara-se com o melhor desempenho, sendo o valor deste relativamente superior aos demais demonstrando a sua importância em relação aos resultados analisados. $O$ gás de proteção $100 \%$ Ar é apontado como o nível capaz de obter o valor máximo para a qualidade do cordão, isso se deve ao fato de que para a mistura $75 \% \mathrm{Ar}+25 \% \mathrm{He}$, contêm hélio, cujo alto potencial de ionização proporciona uma maior tensão do arco elétrico, o que, por sua vez, resulta em maiores aportes térmicos e, com isso, afeta diretamente a qualidade do cordão de solda (SCOTTI et al., 2008).

Na análise da taxa de deposição de arame através do método de Taguchi foi escolhido o padrão "maior é melhor" para razão sinal-ruído, de modo a maximizar a resposta do sistema. A Figura 4 apresenta os resultados referentes a taxa de deposição do arame.

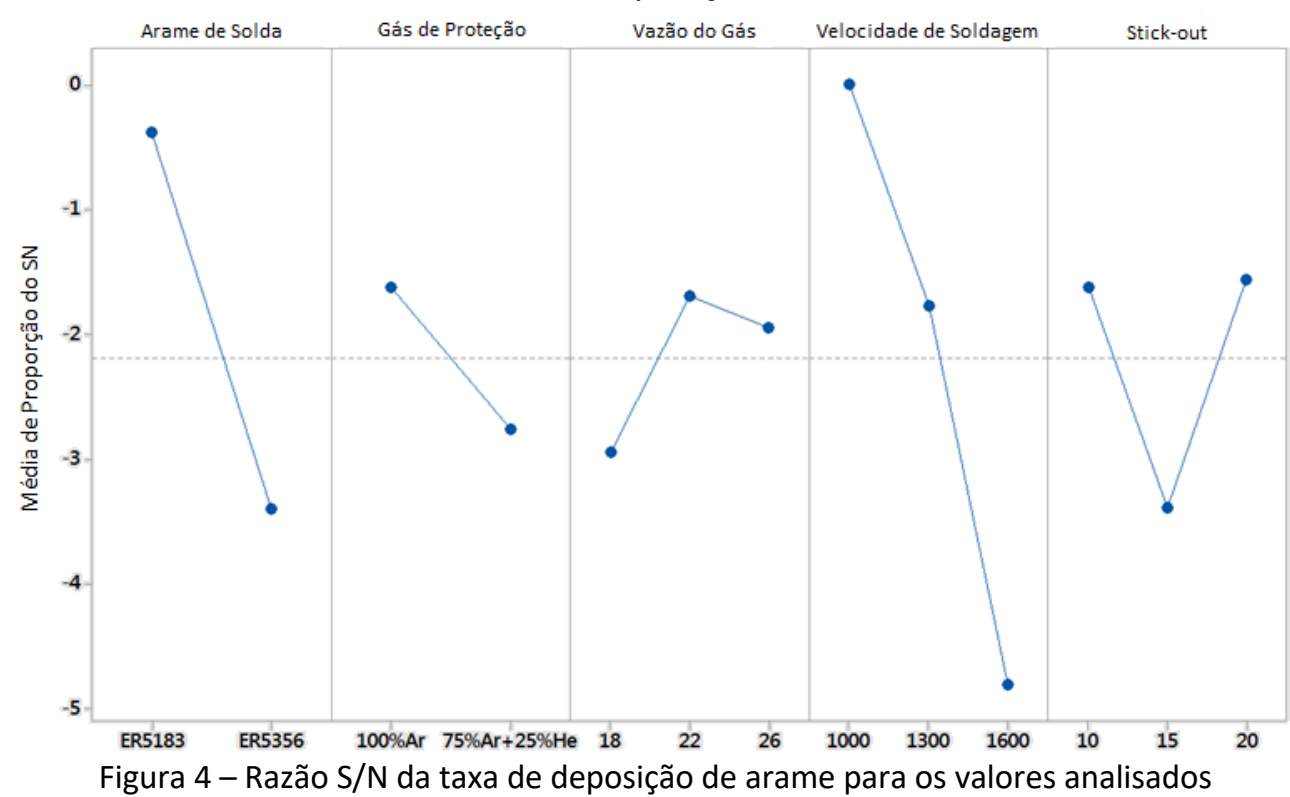

A maior variação do sinal-ruído para a taxa de deposição do arame pode ser verificada pela velocidade de soldagem, sendo este o parâmetro de maior influência na taxa de deposição, visto que maior é a diferença na posição vertical dos pontos marcados (quanto maior o desvio do paralelismo ao eixo-x), maior é a magnitude do efeito. A combinação dos cinco melhores níveis de cada parâmetro que otimizam o processo de soldagem MIG com base nos resultados experimentais para maximizar a variável resposta da taxa de deposição é apresentada na Tabela 6.

Tabela 6 - Melhor configuração de parâmetro de soldagem para a taxa de deposição

\begin{tabular}{c|c|c|c|c}
\hline $\begin{array}{c}\text { Arame de Solda } \\
-\end{array}$ & $\begin{array}{c}\text { Gás de Proteção } \\
-\end{array}$ & $\begin{array}{c}\text { Vazão do Gás } \\
{[\mathrm{L} / \mathrm{min} .]}\end{array}$ & $\begin{array}{c}\text { Velocidade de Soldagem } \\
{[\mathrm{mm} / \mathrm{min} .]}\end{array}$ & $\begin{array}{c}\text { Stick-out } \\
{[\mathrm{mm}]}\end{array}$ \\
\hline ER5183 & 100\%Ar & 22 & 1000 & 20 \\
\hline
\end{tabular}


A maior variação para a razão sinal-ruído foi observada para o parâmetro de velocidade de soldagem, mostrando que este parâmetro tem a maior influência sobre a taxa de deposição. A ordem com que que cada parâmetro selecionado neste estudo afeta a taxa de deposição pode ser classificada como: velocidade de soldagem > arame de solda > stick-out > vazão do gás > gás de proteção.

Barnes (2009), menciona que a taxa de deposição em qualquer processo de soldagem, é o peso do material depositado por unidade de tempo. Os principais fatores que governam a taxa de fusão são o tipo estabilidade da transferência metálica, e ainda as características do fluxo que podem conter elementos de adição. A tensão de soldagem ou tensão do arco é uma variável significativa quanto à geometria da solda, pois afeta o modo de transferência metálica e está associada diretamente ao comprimento do arco (RODRIGUES, 2010). Segundo Souza (2011) a escolha da tensão mais adequada é função da intensidade de corrente (para estabilizar o arco), tipo do arame e do gás de proteção, além da geometria desejada do cordão de solda.

Assim a velocidade de soldagem é um parâmetro que influência na energia de soldagem, e na quantidade de calor transferida à peça, como também na penetração do cordão. Observa-se que a penetração, primeiro aumenta e depois diminui com o aumento da velocidade de soldagem, sendo máxima para velocidades intermediárias. Quando a velocidade é muito baixa, a quantidade de material depositado por unidade de comprimento da solda aumenta, a poça de fusão fica com dimensões elevadas e o calor do arco atua diretamente na poça e não no metal de base, fazendo com que a penetração seja baixa (GOMES, 2006). Já para velocidades de soldagem muito altas, a quantidade de calor transferida por unidade de comprimento é reduzida e a penetração também diminui, podendo ocorrer ainda mordeduras ao longo do cordão (SOUZA, 2011). Desta forma, é imprescindível um correto ajuste da velocidade de soldagem para garantirmos a qualidade da junta soldada.

Na análise do aporte térmico foi escolhido o padrão "menor é melhor", as realizações dos cálculos seguiram as especificações da Equação 2. A Figura 5 apresenta o gráfico do aporte térmico.

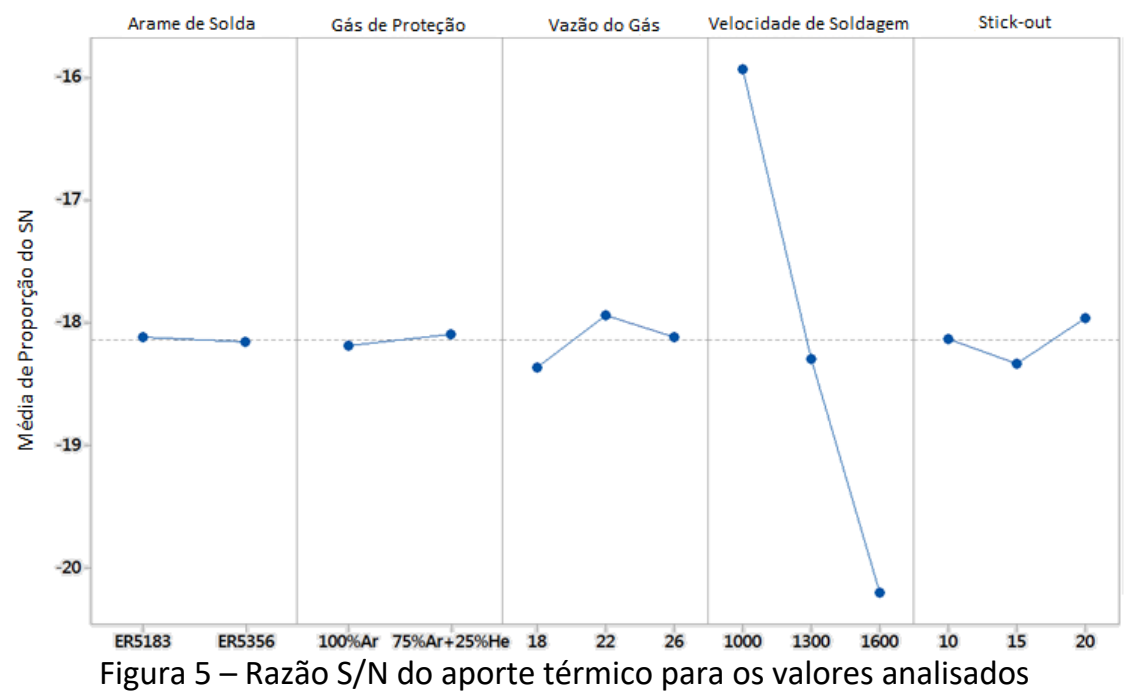

A maior variação do sinal-ruído para o aporte térmico pode ser verificada pela velocidade de soldagem, sendo este o parâmetro de maior influência. A Tabela 7 resume a combinação dos cinco melhores níveis de cada parâmetro para o aporte térmico.

Tabela 7 - Melhor configuração de parâmetro de soldagem para o aporte térmico

\begin{tabular}{c|c|c|c|c}
\hline Arame de Solda & Gás de Proteção & $\begin{array}{c}\text { Vazão do Gás } \\
{[\mathrm{L} / \mathrm{min} .]}\end{array}$ & $\begin{array}{c}\text { Velocidade de Soldagem } \\
{[\mathrm{mm} / \mathrm{min} .]}\end{array}$ & $\begin{array}{c}\text { Stick-out } \\
{[\mathrm{mm}]}\end{array}$ \\
\hline
\end{tabular}


O aporte térmico, ou energia de soldagem, é um dos fatores mais importantes dos processos de soldagem, sendo a velocidade de soldagem o parâmetro que mais possui influência no aporte térmico. A ZTA é a região mais crítica de uma junta soldada, o ciclo térmico pode criar condições para formação de martensita ou estruturas grosseiras. A situação torna-se mais crítica com o aumento do teor de carbono e de elementos de liga do metal base. A soldagem de aços exige alta produtividade, com microestruturas excelentes, porém quanto maior o aporte térmico maior o crescimento do grão e a formação de outras estruturas de baixa propriedade mecânica (CARLOS; CARRITÁ; SOUZA, 2017). Neste estudo a ordem em que cada parâmetro selecionado afeta o aporte térmico pode ser classificada como: velocidade de soldagem > vazão do gás $>$ stick-out $>$ gás de proteção > arame de solda.

De modo que se possa realizar um comparativo entre os resultados do método de Taguchi, os resultados foram compilados e são apresentados na Figura 6.

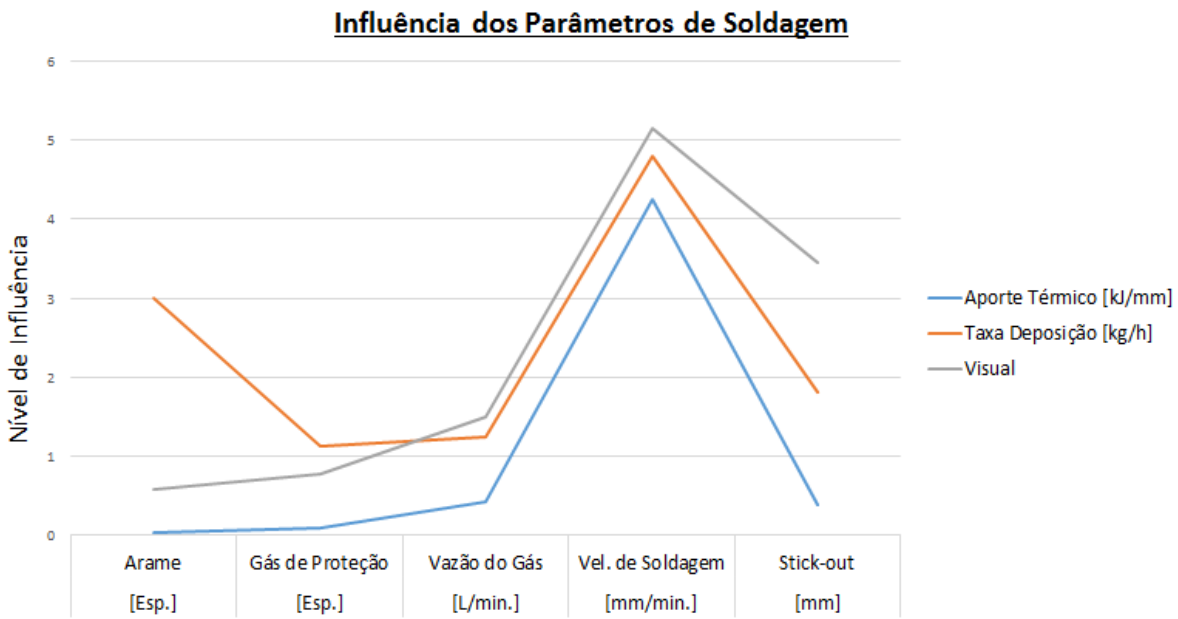

Figura 6 - Comparativo entre a taxa de deposição do arame, aporte térmico e qualidade superficial do cordão de solda.

A Figura 6 apresenta a velocidade de soldagem como sendo o parâmetro que possui maior influência na maximização da resposta para razão sinal-ruído, sendo este crítico para garantir a qualidade e estabilidade do processo MIG. Conclui-se que o método de Taguchi consegue avaliar com segurança as variáveis respostas escolhidas como critério de qualidade.

\section{CONCLUSÕES}

Através da realização de experimentos e da utilização do método Taguchi, detectou-se as influências dos parâmetros de soldagem sobre as variáveis respostas. Os parâmetros das melhores condições são:

- Qualidade superficial do cordão de solda: velocidade de soldagem (1000 mm/min), stickout (10 mm), vazão do gás (18 L/min), tipo de gás de proteção (100\%Ar) e arame de solda (ER5183)

- Taxa de deposição do arame: velocidade de soldagem (1000 mm/min), arame de solda (ER5183), stick-out ( $20 \mathrm{~mm}$ ), vazão de gás (22 L/min) e tipo de gás de proteção (100\%Ar)

- Aporte térmico: velocidade de soldagem $(1000 \mathrm{~mm} / \mathrm{min})$, vazão do gás (22 L/min), stickout $(20 \mathrm{~mm})$, tipo de gás de proteção (75\%Ar25\%He) e tipo de arame de solda (ER5183) 
- Os resultados demostram que a melhor condição para otimizar as variáveis respostas propostas neste estudo é utilizar uma velocidade de soldagem de $1000 \mathrm{~mm} / \mathrm{min}$, stick-out (20 mm), vazão do gás (22 L/min), tipo de gás de proteção (100\%Ar) e arame de solda (ER5183), sendo que o parâmetro de maior influência é a velocidade de soldagem

- O uso do método de Taguchi foi uma ferramenta estatística muito útil, pois permitiu com um número reduzido de ensaios avaliar e selecionar os parâmetros ótimos para obtenção das variáveis respostas desejadas para soldagem MIG.

\section{REFERÊNCIAS}

ALCAN. Alcan Alumínio do Brasil. Manual de Soldagem. Disponível em:< http://mkmouse.com.br/livros/soldaemaluminiomanual-Alcan.pdf>. Acesso em: 15 jun. 2016.

Associação Brasileira de Normas Técnicas. ABNT NBR 6834: alumínio e suas ligas: classificação da composição química. São Paulo: ABNT; 2006.

Associação Brasileira de Normas Técnicas. ABNT NBR 7823: alumínio e suas ligas: chapas propriedades mecânicas. São Paulo: ABNT; 2007.

AWS A5.10/A5.10M:2012 - Welding Consumables - Wire Electrodes, Wires and Rods for Welding of Aluminum Alloys - Classification. American Welding Society, 550N.W. LeJeune Road, Miami, FL 33126 EUA, 2012.

BÚRIGO, B.A.; DE SOUZA, F.G.F.; DUZ, J.L.; MATTIOLI, S.C.; Aquisição do ciclo térmico de soldagem. Faculdade SATC - Departamento de engenharia mecânica. Disponível em: <http://www.ebah.com.br/content/ABAAAAQgOAC/artigo-ciclo-termico-soldagem>. Acesso em: 7 nov. 2016.

BOHRER, C.B. Influência do gás de proteção sobre o aporte de energia na soldagem MIG/MAG de aços ao carbono, Monografia, UFRGS, Porto Alegre, 2013.

GARCIA RP, SCOTT A. Uma metodologia para análises comparativas da capacidade produtiva entre arames maciços (MIG/MAG) e tubulares (Eletrodo Tubular). Soldagem \& Inspeção: 16(2):146-155, 2011.

LIAO, M.T., CHEN, W.J. The effect of shielding gas compositions on the microstructure and mechanical properties of stainless steel weldments. Materials chemistry and physics, v.55, p. 145151, 1998.

MACHADO, I.G. Soldagem \& Técnicas Conexas: Processos, 1a Edição, Porto Alegre, 1996.

MARQUES, P.V., MODENESI, P.J., BRACARENSE, A.Q. Soldagem: fundamentos e tecnologia, 11a ed., Editora UFMG, Belo Horizonte, 2009.

WAINER, B., BRANDI, S.D., MELLO, F.D.H. Soldagem: Processos e Metalurgia, Ed. Edgar Blucher, 1992.

SCOTTI, A. \& PONOMAREV, V. Soldagem MIG/MAG: melhor entendimento, melhor desempenho. 1.ed. Sao Paulo: Artlibre. P. 284, 2008. 
BARNES, G.C. Flux cored arc welding. Chapter 5, DAN TN, 2009.

RODRIGUES, E. R. Influência de parâmetros do processo FCAW-G nas características da solda do aço ASTM A-36. Universidade Tecnológica Federal do Paraná, Fevereiro, 2010.

SOUZA, C. I. Análise comparativa dos processos de soldagem GMAW e FCAW com transferência metálica por curto-circuito na posição horizontal. Dissertação de Mestrado, Universidade Federal de Uberlândia, 2011.

GOMES, E. B. Análise do comportamento da soldagem por curto-circuito aplicado ao processo eletrodo tubular através da metodologia Taguchi. Dissertação de Mestrado, Universidade Federal de Itajubá, Maio, 2006.

CARLOS, J. CARRITÁ, P.H.G. SOUZA, T.I.Z.de. Trabalho de Solidificação -Soldagem. Disponível em http://docplayer.com.br/31320072-Trabalho-de-solidificacao-soldagem-joao-carlos-pedrohenrique-gomes-carrita-taina-itacy-zanin-de-souza.html. Acesso em: 10 jan. 2017. 